\title{
Histone modification-dependent and -independent pathways for recruitment of checkpoint protein Crb2 to double-strand breaks
}

\author{
Li-Lin Du, ${ }^{1}$ Toru M. Nakamura, ${ }^{3}$ and Paul Russell ${ }^{1,2,4}$ \\ ${ }^{1}$ Department of Molecular Biology and ${ }^{2}$ Department of Cell Biology, The Scripps Research Institute, La Jolla, California \\ 92037, USA; ${ }^{3}$ Department of Biochemistry and Molecular Genetics, University of Illinois at Chicago, \\ Chicago, Illinois 60607, USA
}

Cellular responses to DNA damage involve the relocalization of checkpoint proteins to DNA double-strand breaks (DSBs). The fission yeast checkpoint mediator protein Crb2, a homolog of mammalian 53BP1, forms ionizing radiation-induced nuclear foci (IRIF). The IRIF formation by $\mathrm{Crb2}$ requires histone H2A C-terminal phosphorylation and H4-K20 methylation. However, the relevance of Crb2 relocalization is uncertain, because neither histone modification is required for a checkpoint response. Here we show that these histone modifications cooperate in the same Crb2 recruitment pathway, which also requires the Tudor and BRCT motifs in Crb2. In the absence of these histone modifications, an alternative recruitment pathway is sufficient for checkpoint activation and accumulation of Crb2 at a persistent DSB generated by HO endonuclease. This parallel pathway requires a cyclin-dependent kinase phosphorylation site in Crb2 that mediates an association with another BRCT protein Cut5 (the TopBP1 homolog), which also accumulates at HO-induced DSBs. We propose that such dual recruitment mechanisms may be a common feature of DNA damage checkpoint mediators.

[Keywords: Schizosaccharomyces pombe; chromatin; histone code; histone lysine methyltransferase; genome stability; cell cycle]

Supplemental material is available at http://www.genesdev.org.

Received February 21, 2006; revised version accepted April 10, 2006.

The maintenance of genomic integrity relies on a conserved network of proteins that serves surveillance functions, and upon sensing damaged DNA, sets off a concerted response including the arrest of cell cycle progression and the activation of DNA repair. These signaling proteins constitute the DNA damage checkpoint system (McGowan and Russell 2004). In the fission yeast Schizosaccharomyces pombe, residing at the top of the DNA damage checkpoint signaling pathway is the sensor kinase complex Rad3-Rad26 (ATR-ATRIP in mammals) (Furuya and Carr 2003). Rad3-Rad26 phosphorylates and activates the effector kinase Chk1, which then directly controls the activities of cell cycle regulators (Lopez-Girona et al. 2001; Capasso et al. 2002). The phosphorylation of Chk1 by Rad3-Rad26 requires Crb2, which itself is phosphorylated in a Rad3-dependent but Chk1-independent manner upon DNA damage (Saka et al. 1997). This intermediary role of Crb2 correlates with its ability

${ }^{4}$ Corresponding author.

E-MAIL prussell@scripps.edu; FAX (858) 784-2265.

Article is online at http://www.genesdev.org/cgi/doi/10.1101/gad.1422606. to engage in dynamic interactions with $\operatorname{Rad} 3$ and Chk1 (Mochida et al. 2004). Crb2 also interacts with Cut5 (Saka et al. 1997; Mochida et al. 2004). Cut5 is an essential protein with multiple roles in DNA replication and checkpoint signaling (Garcia et al. 2005). The physiological significance of the Crb2-Cut5 interaction remains unclear.

The most conserved structural feature of $\mathrm{Crb} 2$ is a Cterminal BRCT tandem repeat, which resembles similar domains in budding yeast ScRad9 and mammalian 53BP1, BRCA1, and MDC1/NFBD1. These BRCT proteins are each required for the phosphorylation of certain substrates of checkpoint sensor kinases, and collectively have been referred to as adaptors or mediators of the DNA damage checkpoint (Melo and Toczyski 2002; Wang et al. 2002; Canman 2003).

A prominent cellular response to DNA damage is the focal assembly of a large number of DNA repair proteins and checkpoint proteins at double-strand breaks (DSBs) (for review, see Lisby and Rothstein 2005). We have shown before that Crb2 tagged with fluorescent protein forms distinct nuclear foci at DSBs induced by either ionizing radiation (IR) or the site-specific $\mathrm{HO}$ endonucle- 
ase (Du et al. 2003). Efficient formation of IR-induced foci (IRIF) by Crb2 or its mammalian counterparts requires the phosphorylation of histone H2A (H2AX in mammals) at conserved C-terminal SQ sites by checkpoint sensor kinases (Celeste et al. 2003; Nakamura et al. 2004). Despite the dramatic defect in Crb2 IRIF formation, an H2A mutant without the phosphorylation sites displays only mild defects in checkpoint arrest and survival against genotoxic stress (Nakamura et al. 2004). Crb2 IRIF formation also requires the BRCT tandem repeat in $\mathrm{Crb} 2$, but this function of the BRCT repeat is largely dispensable because a leucine zipper dimerization motif can effectively substitute the BRCT repeat for the essential checkpoint function but does not support IRIF formation (Du et al. 2004). These observations suggest that the large-scale accumulation of Crb2 at DSBs is not crucial for its functions. However, it remains possible that a low level Crb2 recruitment still occurs in the H2A and BRCT-truncation mutants.

Recently, another type of histone modification, lysine methylation, has been implicated in checkpoint mediator relocalization. H4-K20 methylation in fission yeast is required for efficient Crb2 IRIF formation (Sanders et al. 2004), whereas H3-K79 methylation is important for 53BP1 relocalization in mammalian cells (Huyen et al. 2004). These findings revealed a new dimension of the increasingly complicated "histone code" linked to DNA damage responses (Downs and Cote 2005; van Attikum and Gasser 2005; Vidanes et al. 2005). These studies have also raised salient questions about the exact mechanisms of checkpoint mediator relocalization. It is unclear whether H4-K20 and H3-K79 methylations are both involved in checkpoint mediator relocalization in the same organism or they represent evolutionary variations between organisms. Furthermore, the fact that mutations affecting H4-K20 methylation in fission yeast do not abolish the functions of Crb2 (Sanders et al. 2004), suggests the possibilities that histone methylations may share overlapping roles with $\mathrm{H} 2 \mathrm{~A}$ phosphorylation, or that there are other unknown mechanisms involved in recruiting checkpoint mediators.

Interestingly, mutations abolishing either $\mathrm{H} 2 \mathrm{~A}$ phosphorylation or H4-K20 methylation displayed synergistic genetic interactions with a hypomorphic crb2 allele, crb2-T215A (Sanders et al. 2004; Nakamura et al. 2005). Crb2-T215 is a cyclin-dependent kinase (CDK) phosphorylation site (Esashi and Yanagida 1999). crb2-T215A mutant cells have a checkpoint maintenance defect ( $\mathrm{Na}$ kamura et al. 2005), and display a moderate sensitivity to IR, which may result from impaired function of topoisomerase Top3 in these cells (Caspari et al. 2002). However, Crb2-T215A forms IRIF normally (Nakamura et al. 2005). The reasons for the genetic interactions between the histone modification mutations and $c r b 2-T 215 \mathrm{~A}$ are unclear.

Here, we further explore the mechanisms of Crb2 recruitment to DSBs by analyzing the phenotypes of fission yeast mutants defective in histone modifications and examining Crb2 relocalization in these mutants using live-cell microscopy and chromatin immunoprecipi- tation (ChIP). We show that H4-K20 but not H3-K79 is required for Crb2 IRIF formation. In the absence of both H4-K20 methylation and H2A phosphorylation, Crb2 remains largely functional, and it can form nuclear focus when a DSB is generated by HO endonuclease. Moreover, we demonstrate that the histone modification-independent accumulation of $\mathrm{Crb} 2$ at $\mathrm{HO}$-induced DSBs requires the Crb2-T215 residue, which mediates a Crb2Cut5 interaction.

\section{Results \\ Histone H4-K2O but not H3-K79 is required for Crb2 IRIF formation}

Efficient IRIF formation by $\mathrm{Crb} 2$ requires $\operatorname{set} 9^{+}$, which encodes the only fission yeast H4-K20 methyltransferase (Sanders et al. 2004). In contrast, the IRIF formation by the Crb2 homolog in vertebrates, 53BP1, depends on $\mathrm{H} 3$ K79 methylation mediated by the Dot1-like methyltransferase (Huyen et al. 2004). The involvement of two different histone methylation sites in the IRIF formation by $\mathrm{Crb} 2$ and 53BP1 has led to the suggestion that perhaps more than one histone methylation event is needed for the recruitment of these checkpoint mediator proteins (Sanders et al. 2004; Stucki and Jackson 2004; Vidanes et al. 2005). To assess whether the conserved H3-K79 residue has a role in Crb2 IRIF formation, we introduced point mutations into a fission yeast strain background in which two of the three $\mathrm{H} 3 / \mathrm{H} 4$ gene pairs have been deleted (Mellone et al. 2003). When an H3-K79A mutation was introduced into this strain, we observed no change in Crb2 IRIF formation compared with wild type (Fig. 1). The previous study by Sanders et al. (2004) did not examine the effect of mutating H4-K20 on Crb2 localization. We found that the H4-K20R mutation dramatically reduced the percentage of nuclei containing Crb2 foci before and after IR treatment, showing that H4-K20 residue is required for Crb2 IRIF formation, as well as spontaneous Crb2 focus formation (Fig. 1). As a control, mutating simultaneously two other conserved lysine residues, H4-K8 and H4-K16, had no effect on Crb2 IRIF formation (Fig. 1). These results confirmed that H4-K20 methylation is required for efficient Crb2 IRIF formation, and ruled out the possibility that H3-K79 methylation is also required for Crb2 IRIF formation.

Crb2 remains functional when its IRIF formation is
compromised by eliminating histone modifications

We next examined whether the absence of strong phenotypes in mutants defective in $\mathrm{H} 2 \mathrm{~A}$ phosphorylation or $\mathrm{H} 4-\mathrm{K} 20$ methylation is due to redundancy between these two types of histone modifications. When both H2A phosphorylation site mutations (hereafter referred to as hta-AQ) and H4-K20R were introduced into the cells of the single $\mathrm{H} 3 / \mathrm{H} 4$ gene background, these cells displayed no stronger sensitivity to UV, hydroxyurea (HU), and IR than the mutants defective in only one type of histone 
A
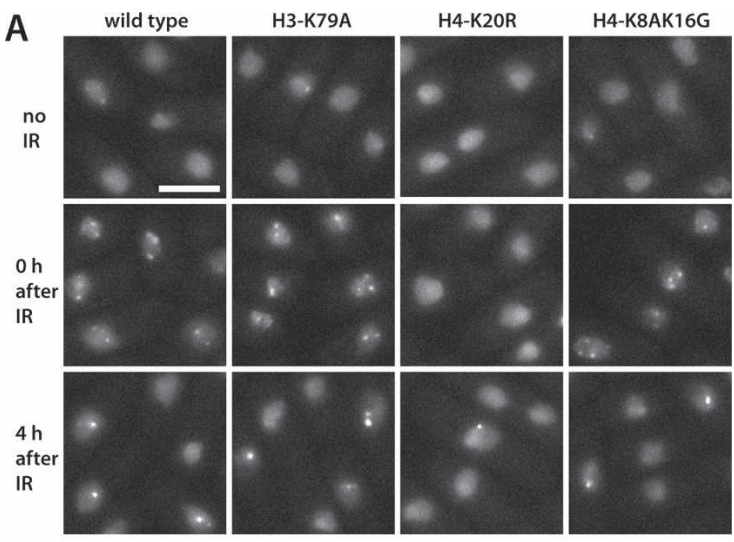

B $\square 1$ focus $\square 2$ foci $\square>=3$ foci

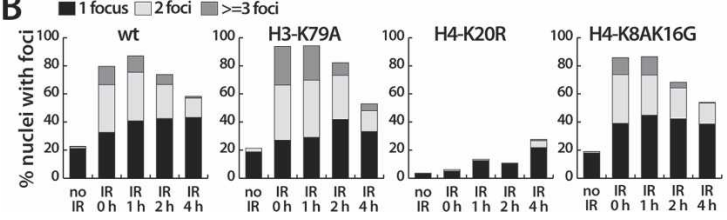

Figure 1. Histone H4-K20 but not $\mathrm{H} 3-\mathrm{K} 79$ is required for efficient Crb2 IRIF formation. (A) H4-K20R mutation dramatically reduced the number of IR-induced Crb2 foci, whereas mutations at three other conserved lysines in histone $\mathrm{H} 3$ and $\mathrm{H} 4$ had no effect. Cells with YFP-tagged Crb2 were treated with a 36-Gy $\gamma$ irradiation and examined by fluorescence microscopy before and at different time points after irradiation. On average, cells receive about four DSBs at this dose (Du et al. 2003). Strains used were LLD3614, LLD3615, LLD3616, and TMN3617. Bar, 5 $\mu \mathrm{m} .(B)$ Quantitation of the microscopy results of the same experiment as in $A$. About 300 nuclei were scored for each time point.

modification (Fig. 2A,B). Furthermore, deleting $\mathrm{crb2}^{+}$in the same strain background generated far more severe phenotypes (Fig. 2A,B), showing that $\mathrm{Crb} 2$ remains largely functional even when both H2A C-terminal phosphorylation and H4-K20 methylation are eliminated.

Compared with normal histone dosage strains, strains harboring a single pair of $\mathrm{H} 3 / \mathrm{H} 4$ genes grow slower and display different sensitivity to genotoxic agents (Sanders et al. 2004; our unpublished results). Therefore, we performed further phenotype analyses in a normal histone dosage background. We used set9s instead of the H4K20R mutation to abolish H4-K20 methylation in this background, as it has been shown that H4-K20 is probably the only Set9 substrate required for the DNA damage response (Sanders et al. 2004). Compared with wild type, both set9 $\Delta$ and $h t a-A Q$ cells displayed mild sensitivities to various genotoxic agents (Fig. 2C,D; Nakamura et al. 2004; Sanders et al. 2004). Interestingly, set9s cells are more sensitive to UV than hta-AQ cells, but hta-AQ cells are more sensitive to the topoisomerase I inhibitor camptothecin (CPT) than set9s cells (Fig. 2C,D), suggesting that $\mathrm{H} 4-\mathrm{K} 20$ methylation and $\mathrm{H} 2 \mathrm{~A}$ phosphorylation have distinct functions in response to different types of DNA lesions despite their common roles in Crb2 IRIF formation. The double mutant of set9 $\Delta$ and hta-AQ exhibited sensitivities similar to the more sensitive single mutant, which were still much less severe than the sensitivities displayed by a $c r b 2 \Delta$ mutant (Fig. 2C,D). A crb2s set9s double mutant showed sensitivities similar to a $c r b 2 \Delta$ mutant. In contrast, hta-AQ partially suppressed the sensitivity of $c r b 2 \Delta$ to IR and UV and enhanced the sensitivity of crb2s to CPT (Fig. 2C,D; Nakamura et al. 2004), suggesting that the functions of H2A phosphorylation are not limited to $\mathrm{Crb} 2$ regulation.

We next analyzed the ability of histone modification mutants to arrest cell cycle progression in response to DNA damage. Cells carrying the cdc25-22 mutation were shifted to restrictive temperature to synchronize them in G2. They were then irradiated by IR while being maintained at restrictive temperature. After IR treatment, cells were released from the G2 block and their progression through mitosis was monitored by microscopy. A crb2s mutant showed no delay of entry into mitosis in response to IR (Fig. 2E). In contrast, the set9 $\Delta$ and hta-AQ single mutants, as well as the set9D hta-AQ double mutant exhibited a substantial delay after IR treatment. As observed before (Nakamura et al. 2004; Sanders et al. 2004), both set9s and hta-AQ mutants entered mitosis slightly earlier than wild type, suggesting that they have a mild checkpoint maintenance defect. No additive checkpoint defect was observed for the set9D hta-AQ double mutant. Taken together, our sensitivity and checkpoint assays demonstrated that Crb2 remains largely functional in the absence of $\mathrm{H} 2 \mathrm{~A}$ phosphorylation and H4-K20 methylation.

\section{A conserved Tudor domain in Crb2 is required for its IRIF formation and acts with Set9 in the same pathway}

We have shown previously that the BRCT repeat in Crb2 is required for efficient IRIF formation (Du et al. 2004). In contrast, the BRCT repeat in $53 \mathrm{BP} 1$ is dispensable for IRIF formation, whereas two tandem Tudor domains upstream of the BRCT repeat are essential for targeting 53BP1 to IRIF (Iwabuchi et al. 2003; Morales et al. 2003; Ward et al. 2003; Charier et al. 2004; Huyen et al. 2004; Pryde et al. 2005). A 56-amino-acid region in the middle of Crb2 sequence shares similarity with the N-terminal Tudor fold of the Tudor tandem repeat in 53BP1 (Supplementary Fig. 1; Huyen et al. 2004; Sanders et al. 2004), suggesting that $\mathrm{Crb} 2$ has at least one Tudor domain. To better understand the structural requirement of Crb2 IRIF formation and the potential roles of the Tudor domain, we expressed yellow fluorescent protein (YFP)tagged Crb2 fragments of different lengths under the control of the $c r b 2^{+}$promoter in a $c r b 2 \Delta$ background and examined their ability to form IRIF (Fig. 3A; Supplmentary Fig. 2). Deleting the N-terminal 354 amino acids, which comprise nearly the entire sequence upstream of the Tudor domain, did not abolish the ability of Crb2 to form IRIF. However, truncating into the Tudor domain by removing another 16 amino acids eliminated IRIF formation. Thus, the Tudor domain in Crb2 is required for 
Du et al.

A

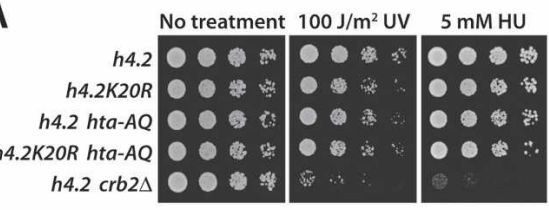

B

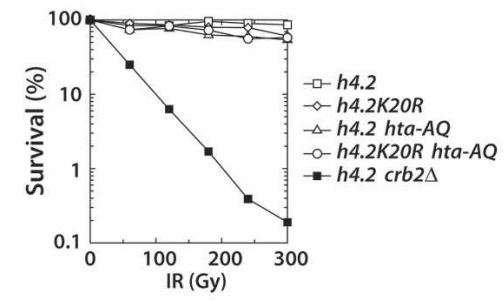

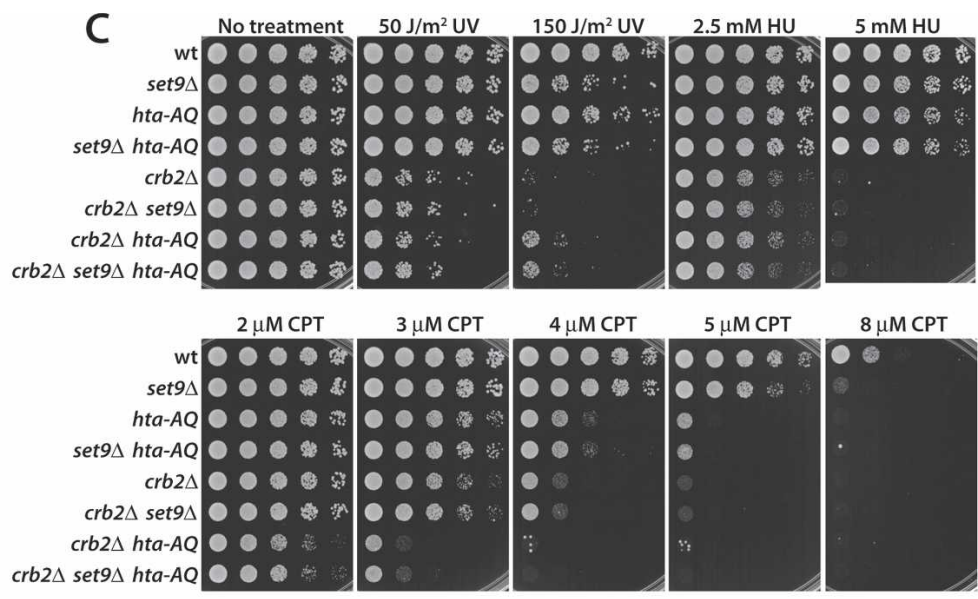

$E$

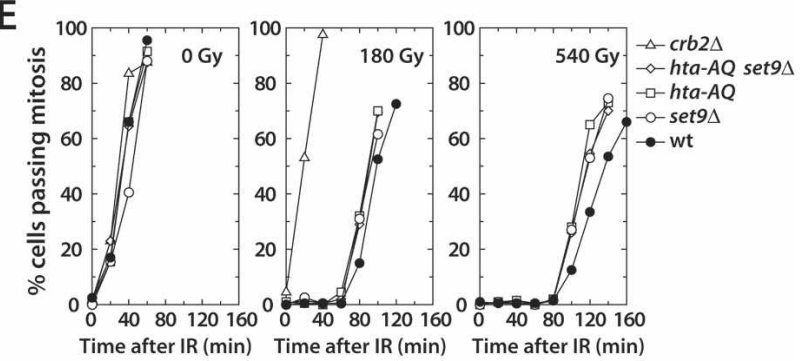

Figure 2. Eliminating both H4-K20 methylation and H2A phosphorylation causes weaker defects in DNA damage responses than $c r b 2 \Delta$. Data shown are representative of multiple experiments. $(A, B)$ H4-K20R mutation (h4.2K20R) and H2A C-terminal phosphorylation site mutations (hta-AQ), either alone or together, resulted in weaker sensitivity to UV, HU, and IR than $c r b 2 \Delta$. Strains were constructed in a genetic background in which two of the three pairs of H3/H4 genes were deleted. Strains used were LLD3618, LLD3619, LLD3620, LLD3621, and LLD3622. (A) Fivefold serial dilutions of cells were spotted on YES plates and incubated at 30 ${ }^{\circ} \mathrm{C}$. Photos were taken $3 \mathrm{~d}$ later for untreated and UV-treated plates. The HU plate was photographed $5 \mathrm{~d}$ later. (B) Survival curves of cells treated with different doses of $\gamma$ irradiation in liquid YES medium. Cells were plated on YES plates after treatment and incubated at $30^{\circ} \mathrm{C}$. The numbers of colonies were counted after $3 \mathrm{~d}$. $(C, D)$ Deleting the H4-K20 methyltransferase gene (set $\left.9 \Delta\right)$ and mutating $\mathrm{H} 2 \mathrm{~A}$ C-terminal phosphorylation sites (hta-AQ), either alone or together, resulted in weaker sensitivity to UV, HU, camptothecin (CPT), and IR than $c r b 2 \Delta$. Strains were constructed in a genetic background with full dosage of H3 and H4 genes. Strains used were LLD3431, LLD3623, TMN3291, LLD3624, LLD3259, LLD3625, LLD3626, and LLD3627. (C) Fivefold serial dilutions of cells were spotted on YES plates and incubated at $30^{\circ} \mathrm{C}$. Plates were photographed $2 \mathrm{~d}$ later, except for the $5-\mathrm{mM}$ HU plate, which was photographed $5 \mathrm{~d}$ later. (D) Survival curves of cells treated with different doses of IR or UV. The numbers of IR-surviving colonies were counted after incubation on YES plates at $30^{\circ} \mathrm{C}$ for $2 \mathrm{~d}$. The numbers of UV-surviving colonies were counted after incubation on YES plates at $30^{\circ} \mathrm{C}$ for $3 \mathrm{~d}$. $(E)$ set $9 \Delta$ and $h t a-A Q$, either alone or together, resulted in a weaker checkpoint defect than crb2s. Strains harboring the cdc25-22 mutation were synchronized at the G2 phase of the cell cycle by shifting to $35.5^{\circ} \mathrm{C}$ for $2.5 \mathrm{~h}$ before IR treatment. After IR treatment, cells were released back to $25^{\circ} \mathrm{C}$ medium. The progression through mitosis was monitored by DAPI and Calcofluor staining. The crb2 $\Delta$ strain was not used in the 540 Gy experiment. Strains used were LLD3628, LLD3629, LLD3630, LLD3631, and LLD3632.

IRIF formation. Neither the BRCT repeat alone, nor any fragment missing the BRCT repeat but containing the Tudor domain, was able to form IRIF, indicating that Crb2 IRIF formation requires the cooperative actions of both the Tudor domain and the BRCT repeat.

To examine the effect of disrupting the Tudor domain in the context of a full-length Crb2 molecule, we mutated a highly conserved phenylalanine residue at position 400 to alanine (Supplementary Fig. 1). When not treated with IR, the YFP-tagged F400A mutant displayed diffuse nuclear fluorescence with intensity comparable to that of wild-type protein (our unpublished results). However, IRIF formation was largely abolished by the F400A mutation (Fig. 3B). Therefore, the conserved F400 residue in the Tudor domain is necessary for IRIF formation.
When we introduced the F400A mutation into the endogenous crb2 gene, cells became mildly sensitive to UV, CPT, and HU (Fig. 3C). Because the Tudor tandem repeat in 53BP1 was shown to interact with K79 methylated histone H3 (Huyen et al. 2004), we hypothesized that the Tudor domain in Crb2 may be involved in interactions with $\mathrm{K} 20$ methylated histone $\mathrm{H} 4$. To probe the functional connection between the Tudor domain and H4-K20 methylation, we examined the relationship between crb2-F400A and set9A (Fig. 3C). crb2-F400A was slightly more sensitive to UV and CPT than set9 $\Delta$. The double-mutant crb2-F400A set9s displayed the same sensitivity as crb2-F400A. Thus, crb2-F400A affects the same pathway as set $9 \Delta$. Another hypomorphic mutation of $c r b 2$, crb2-T215A, is known to synergistically interact with set9s and hta-AQ (Sanders et al. 

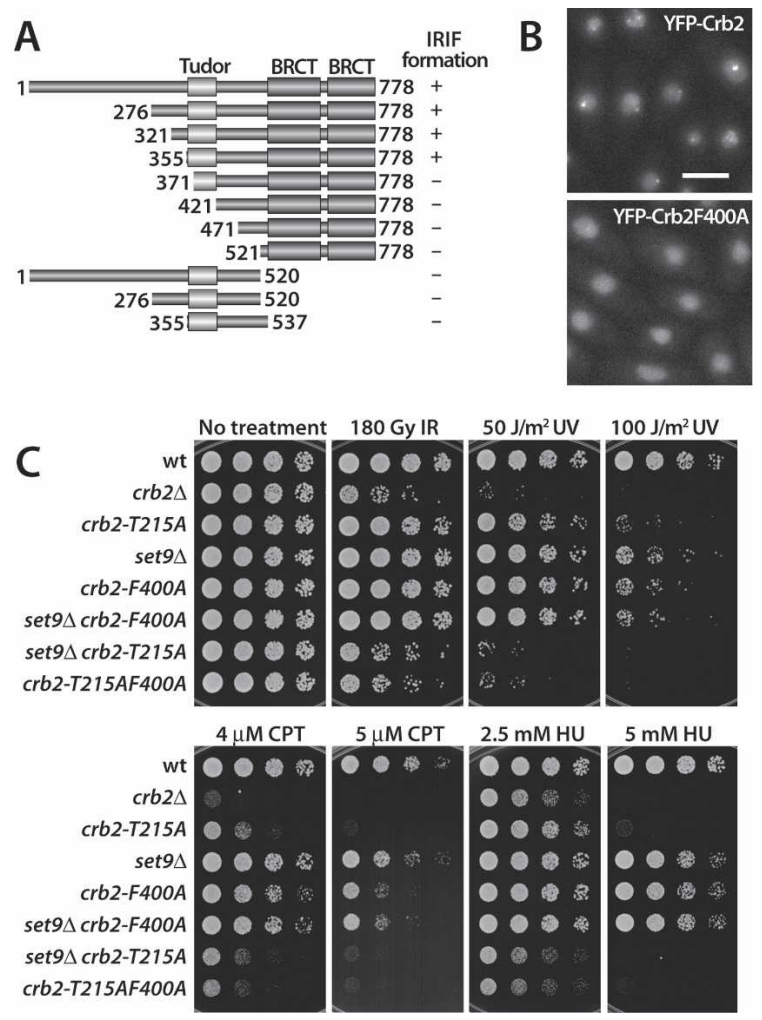

Figure 3. A conserved Tudor domain in Crb2 is necessary for efficient Crb2 IRIF formation and a Tudor domain mutation affects the same pathway as set $9 \Delta$ with regard to sensitivities to genotoxic agents. (A) Two conserved regions of Crb2-a Tudor domain in the middle of the protein and a C-terminal BRCT tandem repeat-are each necessary for efficient IRIF formation. See Supplementary Figure 2 for images. Truncated Crb2 fragments were fused at their $\mathrm{N}$ terminus with YFP and expressed from the $c r b 2^{+}$promoter in a crb2 2 strain. (B) Mutating a conserved phenylalanine residue in the Tudor domain largely abolished Crb2 IRIF formation. Cells were treated with a 36-Gy $\gamma$ irradiation and observed by fluorescence microscopy immediately after treatment. Strains used were LLD3642 and LLD3643. Bar, $5 \mu \mathrm{m} .(C)$ The crb2-F400A mutation caused milder sensitivity to genotoxic agents than $c r b 2 \Delta$, exhibited no additive phenotype when combined with set $9 \Delta$, and enhanced the genotoxic sensitivity of the crb2-T215A mutant in a fashion similar to set9 . Fivefold serial dilutions of cells were spotted on YES plates and incubated at $30^{\circ} \mathrm{C}$. IR treatment was applied to liquid cultures and cells were then spotted on plates. Plates were photographed $2 \mathrm{~d}$ later, except for the 5-mM HU plate, which was photographed 3 d later. Strains used were LLD3431, LLD3423, LLD3644, LLD3645, LLD3646, LLD3647, LLD3648, and LLD3649.

2004; Nakamura et al. 2005). T215 is a CDK phosphorylation site (Esashi and Yanagida 1999). We found that combining the T215A and F400A mutations also led to a strong additive effect and resulted in genotoxic sensitivities approaching the level of $c r b 2 \Delta$ (Fig. 3C). Taken together, these results suggest that histone modifications and the Tudor domain act in the same pathway to regulate Crb2, whereas the Crb2-T215 residue is involved in a parallel pathway. Furthermore, the lack of an additive effect when combining crb2-F400A and set9s strongly suggests that the main role of histone H4-K20 methylation is regulating Crb2 function.

\section{Crb2 can be recruited to an HO-induced DSB in the absence of histone H2A phosphorylation and H4-K20 methylation}

That Crb2 remains largely functional in histone modification mutants led us to hypothesize that Crb2 may be recruited to sites of DNA damage through histone modification-independent mechanisms, which accumulate Crb2 at sites of IR-induced DNA damage to a level below the detection limit of fluorescence microscopy, but nonetheless sufficient for checkpoint activation. To explore this possibility we turned to an alternative method for detecting protein relocalization on chromatin, the ChIP assay.

To generate specific DSBs in fission yeast cells for ChIP analysis we used the site-specific HO endonuclease (Haber 2002). We have shown previously that HO-induced DSBs resulted in the nuclear focus formation by Crb2 and Rad22 (a Rad52 homolog) (Du et al. 2003). HOinduced Crb2 foci and Rad22 foci colocalize with each other and with a lacO marker placed next to the $\mathrm{HO}$ cleavage site (Du et al. 2003). In the present study, we used the strain harboring a single $\mathrm{HO}$ cleavage site at the arg3 locus. To prevent variable $\mathrm{HO}$ expression due to the change of plasmid copy number, we integrated the plasmid expressing $\mathrm{HO}$ under the control of a thiamine-repressible promoter into the chromosome. In addition, Crb2 was tagged with the tandem affinity purification (TAP) tag (Rigaut et al. 1999) to facilitate immunoprecipitation with IgG beads, and $\operatorname{Rad} 22$ was tagged with cyan fluorescence protein (CFP) to visually monitor the induction of DSBs. The derepression of the thiaminerepressible promoter requires cells to be grown in the absence of thiamine for at least five doubling times (Tommasino and Maundrell 1991). With our HO system it takes $\sim 22 \mathrm{~h}$ at room temperature to see an appreciable increase of Rad22-CFP foci after the removal of thiamine from the medium (our unpublished observation). Using PCR with a pair of primers flanking the HO cleavage site, we determined that $\sim 80 \%$ of the cells received a DSB $23 \mathrm{~h}$ after the removal of thiamine (Fig. 4A). Thus, we used cells grown in the absence of thiamine for $23 \mathrm{~h}$ and cells grown in the presence of thiamine for ChIP analysis. DNA fragments coprecipitated with TAP-Crb2 were examined by multiplex PCR. Four pairs of PCR primers annealing to regions $0.2,2,9$, and $16 \mathrm{~kb}$ away from the $\mathrm{HO}$ cleavage site were used to assess the binding of Crb2 to DNA adjacent to the HO-induced DSB (Fig. 4B). One additional pair of primers that anneal to the act 1 gene on a different chromosome was used as an internal control. HO induction resulted in substantial increase of coprecipitated DNA at sites $0.2,2$, and $9 \mathrm{~kb}$ from the HO cleavage site in wild-type cells (Fig. 4C). A weaker but reproducible increase was also detected at the $16-\mathrm{kb}$ site. Thus, Crb2 accumulation on chromatin spreads from the immediate vicinity of the $\mathrm{HO}$-induced 
Du et al.

Figure 4. H4-K20 methylation, H2A C-terminal phosphorylation, and the conserved motifs in Crb2 are not required for recruiting $\mathrm{Crb} 2$ to an HO-induced DSB. (A) Expression of $\mathrm{HO}$ endonuclease in a strain harboring a single HO cleavage site generated DSBs in the majority of the cells. Twofold serial dilutions of genomic DNA were used as template for PCR with a pair of primers annealing to regions flanking the HO cleavage site. PCR amplifying from the unrelated act1 locus was used as control. $(B, C)$ ChIP analysis demonstrated that TAP-tagged Crb2 is recruited to chromatin regions adjacent to the HO-induced DSB in both wild-type and the histone modification mutants. (B) Locations of the PCR primer pairs used for amplifying DNA regions adjacent to the $\mathrm{HO}$ cleavage site. $(C)$ DNA from the input $(-0.003 \%$ of total) and the IgG bead-bound fraction $(13 \%$ of total) was analyzed by multiplex PCR. Strains expressing YFP-Crb2 served as control for nonspecific binding of DNA to the IgG beads. Strains used were LLD3652, LLD3650, LLD3716, LLD3717, and LLD3718. (D) Eliminating either or both H4-K20 methylation and H2A C-terminal phosphorylation did not abolish HO-induced YFP-Crb2 nuclear focus formation. CFP-tagged Rad22 expressed in the same cells was used as an independent marker for HO-induced DSBs. Merges were overlays of fluorescence images (YFP in red, CFP in green) and bright-field image of the same field. Strains used were LLD3650, LLD3651, LLD3652, and LLD3653. Bar, 5 um. $(E, F)$ HO-induced Crb2 nuclear focus formation can occur independent of the Tudor domain and the BRCT tandem repeat. (E) F400 residue in the Crb2 Tudor domain is dispensable for the HO-induced focus formation by full-length Crb2 protein, but is required for the HO-induced focus formation by Crb2(355-778). CFP-tagged Rad22 expressed in the same cells was used as an independent marker for HO-induced DSBs. Strains used were LLD3654, LLD3655, and LLD3656. (F) An N-terminal fragment of Crb2 missing both the Tudor domain and the BRCT tandem repeat, Crb2(1-358), when fused with a leucine zipper motif, could form HO-induced foci in the absence of H4-K20 methylation and H2A C-terminal phosphorylation. The strain used was LLD3658.

DSB to regions at least several kb away. Surprisingly, a similar HO-induced increase of Crb2-bound DNA was found in $h t a-A Q$ and set $9 \Delta$ cells (Fig. 4C). The binding of Crb2 to DNA in wild-type and the histone modification mutants presumably happens in different chromatin contexts. Thus, there may be differences in formaldehyde cross-linking efficiency between these strains, which would complicate any attempt to compare enrichment ratios. Despite this limitation, we can conclude that $\mathrm{Crb} 2$ is enriched on chromatin adjacent to the HOinduced DSB in hta-AQ and set9D mutants.

The wild-type-like enrichment of $\mathrm{Crb} 2$ at an $\mathrm{HO}$-induced DSB in histone modification mutants as revealed by the ChIP assay prompted us to reevaluate the ability of microscopy to detect Crb2 accumulation in histone modification mutants. We surmised that a persistent DSB generated by HO may lead to a level of Crb2 accumulation high enough to be detected by live-cell imaging even in histone modification mutants. Indeed, this sup- position was correct. HO expression in cells harboring a single $\mathrm{HO}$ cleavage site resulted in the formation of a distinct Crb2 nuclear focus in a majority of the wildtype, hta-AQ, set9s, and hta-AQ set9s cells (Fig. 4D). Most of the HO-induced Crb2 foci colocalized with $\mathrm{HO}$ induced Rad22 foci. Therefore, microscopic observations independently confirmed the ChIP results and further demonstrated that $\mathrm{Crb} 2$ can be recruited to an HO-induced DSB in the absence of both H2A phosphorylation and H4-K20 methylation.

\section{Crb2 can be recruited to an HO-induced DSB through its $N$-terminal region}

The ability to visualize Crb2 recruitment to an $\mathrm{HO}$-induced DSB in hta-AQ and set9s cells encouraged us to assess $\mathrm{Crb} 2$ relocalization in other mutants with the $\mathrm{HO}$ system. The Crb2-F400A mutation, which abolished Crb2 IRIF formation, did not affect the formation of $\mathrm{HO}$ - 
induced Crb2 foci (Fig. 4E), suggesting that the Tudor domain is dispensable for recruiting $\mathrm{Crb} 2$ to $\mathrm{HO}$-induced DSBs. Interestingly, the F400 residue became essential for the formation of HO-induced foci when the N-terminal region upstream of the Tudor domain was truncated (Fig. 4E). Thus, there appear to be two redundant mechanisms of forming $\mathrm{HO}$-induced $\mathrm{Crb} 2$ foci, with the $\mathrm{N}$ terminal region of $\mathrm{Crb} 2$ required for one pathway and the Tudor domain required for the other.

The essential function of the BRCT repeat in Crb2 is to mediate homo-oligomerization (Du et al. 2004). This oligomerization role of the BRCT repeat is conserved in budding yeast ScRad9 (Soulier and Lowndes 1999). The BRCT repeat in Crb2 can be effectively substituted by a 32-amino-acid leucine zipper dimerization motif (Du et al. 2004). The leucine zipper fusion, Crb2(1-520)-LZ, is largely functional in checkpoint activation and genotoxic resistance even though it does not form IRIF. In light of the results presented above, we examined the ability of BRCT-truncated $\mathrm{Crb} 2$ fragments to form HOinduced foci. Crb2(1-520)-LZ efficiently formed HO-induced foci, whereas Crb2(1-520) failed to form HO-induced foci (Supplementary Fig. 3), indicating that the dimerization of $\mathrm{Crb} 2$ is required for its accumulation at HO-induced DSBs. Because mutating the conserved F400 residue in the Tudor domain did not result in strong damage sensitivity, nor did it abolish $\mathrm{HO}$-induced focus formation, we reasoned that the only essential function of the C-terminal 420 amino acids of Crb2 that contain the Tudor and BRCT motifs is to mediate homo-oligomerization. Hence, we constructed a leucine zipper fusion with the $\mathrm{N}$-terminal region of $\mathrm{Crb} 2$ upstream of the Tudor domain, Crb2(1-358)-LZ. Remarkably, this fusion protein is largely functional. It strongly rescued the sensitivity of $c r b 2 \Delta$ to IR, UV, and HU (Fig. 5D). Furthermore, it efficiently formed HO-induced foci in the histone modification mutant and wild-type cells (Fig. 4F; our unpublished observations). Therefore, when provided a means to dimerize, the $\mathrm{N}$-terminal region of
Crb2 can be efficiently recruited to an HO-induced DSB independent of histone $\mathrm{H} 2 \mathrm{~A}$ phosphorylation and $\mathrm{H} 4$ K20 methylation. Even though it is formally possible that other currently unknown histone modifications are involved in the Crb2 recruitment pathway independent of H2A phosphorylation and H4-K20 methylation, for simplicity we will refer to this pathway as the histone modification-independent recruitment pathway. We next asked what is required for this histone modification-independent recruitment pathway.

\section{Crb2-T215 residue is required for histone modification-independent recruitment of Crb2 to DSBS}

The strong synergistic effect of combining the Crb2 Nterminal truncation and the F400A mutation on HOinduced focus formation (Fig. 4E) correlated with genetic interactions between crb2-T215A and crb2-F400A in DNA damage sensitivity assays (Fig. 3C). Furthermore, the crb2-T215A mutation is known to exhibit strong synthetic interactions with both set9 $\Delta$ and $h t a-A Q$ in checkpoint and genotoxin survival assays (Sanders et al. 2004; Nakamura et al. 2005), indicating that the T215 residue is required in a pathway parallel to that mediated by histone modifications. Therefore, we examined whether T215 is needed for the histone modificationindependent accumulation of $\mathrm{Crb} 2$ at $\mathrm{HO}$-induced DSBs. The Crb2-T215A mutation by itself has no effect on Crb2 IRIF formation (Nakamura et al. 2005), nor does it affect HO-induced Crb2 focus formation (Fig. 5A). However, when expressed as the only Crb2 in set9s and htaAQ mutant cells, Crb2-T215A failed to form HO-induced foci, even though Rad22 foci were induced normally in these cells (Fig. 5A). In addition, these cells failed to arrest in response to HO-induced DNA breaks, as many of them underwent nuclear division with persisting Rad22 foci, which very rarely happens in wildtype cells (Fig. 5A). The T215 residue was also found to

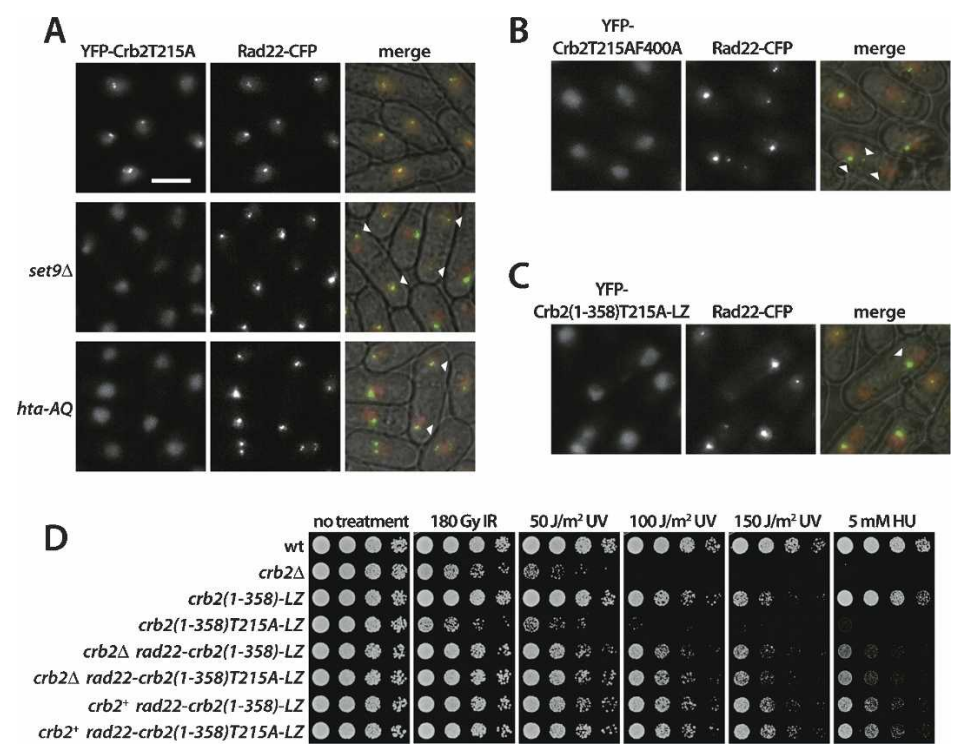

Figure 5. Histone modification-independent relocalization of $\mathrm{Crb} 2$ to DSBs requires the Crb2-T215 residue. (A) HO-induced focus formation by YFP-Crb2T215A was abolished by set $9 \Delta$ or hta-AQ mutations. Strains used were LLD3659, LLD3660, and LLD3661. Arrowheads indicate Rad22 nuclear foci in cells undergoing nuclear division. Bar, $5 \mathrm{um}$. (B) T215 is required for $\mathrm{HO}$-induced focus formation by Crb2F400A. The strain used was LLD3662. (C) T215 is required for HO-induced focus formation by Crb2(1-358)-LZ. The strain used was LLD3663. (D) T215A mutation rendered Crb2/1358)-LZ defective in conferring resistance to DNA damage, and this defect could be rescued by fusing the Crb2 fragment to Rad22. Fivefold serial dilutions of cells were spotted on YES plates and incubated at $30^{\circ} \mathrm{C}$. IR treatment was applied to liquid cultures and cells were then spotted on plates. Plates were photographed 2 d later, except for the 5-mM HU plate, which was photographed 3 d later. Strains used were LLD3431, LLD3423, LLD3666, LLD3667, LLD3668, LLD3669, LLD3670, and LLD3671. 
be critical for the formation of HO-induced foci by Crb2F400A and Crb2(1-358)-LZ, as well as their ability to enforce checkpoint arrest (Fig. 5B,C). The defects of these mutants in focus formation and checkpoint arrest were not due to abnormal expression levels, because these mutants displayed diffuse nuclear signal similar to that of wild-type protein in cells without foci (our unpublished observation).

We summarize in Table 1 our microscopy observations of histone modification mutants and crb2 mutants together with the IR-sensitivity of these strains. The histone modification mutants and the $\mathrm{Crb} 2$ mutants defective in the conserved domains share the same IRIF formation defect, but they are similarly competent in three functional aspects: HO-induced Crb2 focus formation, checkpoint arrest in response to an HO-induced DSB, and resistance to IR. However, the same mutants completely lose all these functions when the T215A mutation is introduced, even though the T215A mutation by itself does not generate a strong phenotype.

Taken together, the microscopy and genetic evidence led us to conclude that Crb2 is recruited through two parallel pathways: One is mediated by histone modifications and the conserved motifs in Crb2, whereas the other is dependent on the T215 residue.

The critical requirement of the T215 residue for the histone modification-independent formation of HO-in- duced Crb2 foci suggests that the essential role of T215 in the absence of histone modifications is to recruit $\mathrm{Crb} 2$ to sites of DNA damage. To further confirm this hypothesis, we examined whether relocalizing $\mathrm{Crb} 2$ to sites of DNA damage through an independent means can bypass the requirement of T215. We constructed a fusion between Crb2(1-358)T215A-LZ, which is defective in both Crb2 recruitment pathways and does not rescue crb2s, and Rad22, which can accumulate at sites of DNA damage independent of Crb2. Cells that expressed the fusion protein as their only form of Rad22 did not exhibit the severe slow growth phenotype of rad22 $\Delta$ cells, indicating that Rad22 functions were not grossly compromised by the fusion (our unpublished observation). Furthermore, the fusion protein could strongly rescue the IR and UV sensitivity of crb2 $\Delta$ (Fig. 5D). In fact, it conferred the same level of resistance to DNA damage as the fusion protein between Crb2(1-358)-LZ and Rad22, suggesting that the severe defects of Crb2(1-358)T215A-LZ were not due to a loss of the intrinsic ability of this molecule to induce checkpoint signaling, and T215 is no longer required when an alternative means of recruitment is provided. Therefore, we conclude that in the absence of the histone modification-dependent recruitment pathway, the main role of the T215 residue in promoting resistance to DNA damage is the recruitment of Crb2 to sites of DNA damage.

Table 1. The effects of crb2 mutations and histone modification mutations on DNA damage-induced Crb2 focus formation, checkpoint activation, and resistance to IR

\begin{tabular}{|c|c|c|c|c|}
\hline Strains & $\begin{array}{l}\text { IRIF in } \\
\text { asynchronous cells }\end{array}$ & $\begin{array}{l}\text { HO-induced } \\
\text { Crb2 foci }\end{array}$ & $\begin{array}{l}\text { Arrest upon } \\
\text { HO induction }\end{array}$ & $\begin{array}{l}\text { Resistance } \\
\text { to IR }\end{array}$ \\
\hline Wild type & + & + & + & + \\
\hline crb2T215A & + & + & + & + \\
\hline crb2(355-778) & + & + & - & - \\
\hline $\operatorname{set} 9 \Delta$ & - & + & + & + \\
\hline hta-AQ & - & + & + & + \\
\hline set9 9 ta-AQ & - & + & + & + \\
\hline $\operatorname{crb} 2 F 400 A$ & - & + & + & + \\
\hline$c r b 2(1-358)-L Z$ & - & + & + & + \\
\hline crb2(1-358)-LZ set9D hta-AQ & - & + & + & + \\
\hline $\operatorname{crb2}(1-520)-L Z$ & - & + & + & + \\
\hline crb2T215A set9s & - & - & - & - \\
\hline crb2T215A hta-AQ & - & - & - & - \\
\hline crb2T215AF400A & - & - & - & - \\
\hline crb2(1-358)T215A-LZ & - & - & - & - \\
\hline crb2(355-778)F400A & - & - & - & - \\
\hline crb2(1-520) & - & - & - & - \\
\hline
\end{tabular}

aplus sign $(+)$ indicates that the majority of the nuclei $(>50 \%)$ displayed at least one Crb2 focus immediately after a 36-Gy treatment; before treatment, $20 \%$ of these nuclei had spontaneous Crb2 foci. Minus sign (-) indicates that a very small number of nuclei $(<10 \%)$ displayed Crb2 foci immediately after a 36-Gy treatment; these strains also had a very low frequency of spontaneous Crb2 foci without treatment.

${ }^{b} \mathrm{Rad} 22-\mathrm{CFP}$ focus formation was used to monitor the HO-induced DSB formation. Plus sign (+) indicates that the majority of the Rad22 foci $(>50 \%)$ colocalized with Crb2 foci. Minus sign (-) indicates that Crb2 focus was undetectable.

${ }^{\mathrm{c}}$ Rad22-CFP focus formation was used to monitor the HO-induced DSB formation. Plus sign $(+)$ indicates that the most of the cells with Rad22-CFP foci became elongated and failed to progress into mitosis. Minus sign (-) indicates the lack of cell elongation and the occurrence of binuclear cells with Rad22-CFP foci.

${ }^{\mathrm{d}}$ Plus sign (+) indicates high level of survival (>70\%) after a 100-Gy treatment. Minus sign (-) indicates survival rates similar to crb2 $\Delta$ $(<10 \%)$ after a 100-Gy treatment. Data were from this study and previous publications (Du et al. 2004; Nakamura et al. 2004, 2005; Sanders et al. 2004). 
Persistent IR-induced DSBs result in Crb2 focus formation independent of histone modifications

It is apparent from Table 1 that HO-induced focus formation correlates better than IRIF formation with the functional capacity of Crb2 in checkpoint induction and promoting survival against IR. We propose that the difference between IR and HO-induced focus formation lies in the unrepairable nature of the HO-induced DSBs (see Discussion). The persistent HO-induced DSBs allow a much higher level of Crb2 accumulation in mutants that fail to form visible IRIF. Our hypothesis would predict that if persistent DSBs are generated by IR, Crb2 accumulation at such DSBs will mimic the situations of HOinduced DSBs. Homologous recombination is the predominant pathway for DSB repair in fission yeast (Manolis et al. 2001; Ferreira and Cooper 2004). Most fission yeast cells in an asynchronous culture are in G2 phase of the cell cycle, and therefore, can easily repair an IR-induced DSB with homologous sequence on the sister chromatid. To restrict the availability of homologous templates for recombination repair, we synchronized cells in early $\mathrm{S}$ phase with HU before IR treatment (Supplementary Fig. 4). These cells suffer persistent DSBs, as evidenced by the large numbers of Rad22 foci 6 $\mathrm{h}$ after treatment (Supplementary Figs. 4, 5). Consistent with our prediction, Crb2 foci were observed in nearly $40 \%$ of the hta-AQ set $9 \Delta$ cells $6 \mathrm{~h}$ after treatment, whereas Crb2-T215A failed to form foci in the set9A background (Supplementary Figs. 4, 5). Also consistent with our model was the observation that a recombination repair mutant $(r h p 51 \Delta)$ has a high level of spontaneous and IR-induced $\mathrm{Crb} 2$ foci in the absence of H2A phosphorylation (Supplementary Fig. 6).

Cut5 is important for histone

modification-independent recruitment of Crb2 to DSBs

We next asked how T215 is involved in the histone modification-independent recruitment of Crb2. The only protein known to interact with the $\mathrm{N}$-terminal region of Crb2 is the BRCT protein Cut5 (Saka et al. 1997; Esashi et al. 2000; Du et al. 2004; Mochida et al. 2004). Cut5 is homologous to budding yeast Dpb11 and mammalian TopBP1, and is essential for DNA replication and both the replication and DNA damage checkpoint signaling pathways (Garcia et al. 2005). The Crb2-binding region in Cut 5 contains the first two of its four BRCT domains (Saka et al. 1997). BRCT domains are phosphopeptidebinding modules (Manke et al. 2003; Yu et al. 2003). In fact, one study has demonstrated that the first two BRCT domains in Cut5 preferentially bind phosphopeptides in a peptide library (Yu et al. 2003). Because the Crb2-T215 residue is phosphorylated in vivo, we considered whether it plays a role in the Crb2-Cut5 interaction. Using a yeast two-hybrid assay, we found that the amino acid sequence in Crb2 between positions 211 and 245 is required for the Crb2-Cut5 interaction (Fig. 6A). Furthermore, the Crb2-T215A mutation strongly diminished this interaction (Fig. 6A,B). Thus, T215 is critical for the association between $\mathrm{Crb} 2$ and Cut5. Consistent with the possibility that the Crb2-Cut5 interaction may require the phosphorylation of Crb2-T215, replacing T215 with acidic residues that mimic constitutively phosphorylated threonine did not compromise the Crb2-Cut5 interaction as much as T215A (Fig. 6A,B). We have shown before that, in vivo, these phospho-mimetic mutations resulted in weaker DNA damage sensitivity than T215A (Nakamura et al. 2005).

If the Crb2-Cut5 interaction mediated by the Crb2T215 residue is required for the histone modificationindependent relocalization of $\mathrm{Crb2}$, we expected that Cut5 should localize at an HO-induced DSB. To test this idea, we tagged Crb2 with YFP and Cut5 with CFP and monitored their subnuclear distribution by live-cell imaging. When HO-induced DSBs were generated in cells harboring a single $\mathrm{HO}$ cleavage site, similar focal assembly occurred with Crb2 and Cut5. Both Crb2 and Cut5 formed a single bright nuclear focus in most cells and their foci colocalized with each other (Fig. 6C). Twentysix hours after $\mathrm{HO}$ induction, $89 \%$ of the $\mathrm{Crb} 2$ foci overlapped with Cut 5 foci $(n=93)$, and $93 \%$ of the Cut 5 foci overlapped with Crb2 foci $(n=89)$. In contrast, Crb2 and Cut5 showed different types of localization after a 36-Gy IR treatment. Crb2 foci were readily detectable in most cells, whereas Cut5 foci occurred much less frequently than Crb2 foci, and the IR-induced Cut5 foci were also much fainter than the HO-induced Cut5 foci (Fig. 6D). Even when all the faint Cut5 foci were counted, only $15 \%$ of Crb2 foci colocalized with Cut5 foci after IR treatment $(n=247)$, whereas $92 \%$ of Cut5 foci colocalized with Crb2 foci $(n=39)$. Therefore, the accumulation of Cut5 at sites of DNA damage is the strongest under conditions that permit the microscopic detection of histone modification-independent $\mathrm{Crb} 2$ relocalization. This pattern of Cut5 localization is consistent with the idea that chromatin-bound Cut5 at the sites of DNA damage facilitates histone modification-independent $\mathrm{Crb} 2$ recruitment. Furthermore, we found that HO-induced Cut5 focus formation occurs independent of histone modifications and Crb2 (Supplementary Fig. 7). Cut5 accumulation at the sites of DSBs may be mediated by the interactions between its C-terminal BRCT repeat and the PCNA-like checkpoint protein Rad9 (Furuya et al. 2004).

To directly assess the role of Cut 5 in histone modification-independent Crb2 relocalization, we employed a temperature-sensitive allele of cut5, cut5-T401, which harbors mutations that result in two amino acid changes, T45M and K62Q (Saka et al. 1997). The T45M mutation impairs the two-hybrid interaction between Cut5 and Crb2 (Saka et al. 1997). We caution here that to properly interpret data obtained with cut5-T401, one has to take into consideration the hypomorphic nature of the mutant allele and its pleiotropic defects in other checkpoint signaling events (Saka et al. 1994; Harris et al. 2003; Furuya et al. 2004). At room temperature, cut5T401 and set9D mutants displayed normal HO-induced Crb2 focus formation (Fig. 6F,G). However, the double- 
Du et al.

Figure 6. Cut5 is involved in the histone modification-independent relocalization of Crb2 to DSBs. $(A, B)$ The Crb2-T215 residue is important for an interaction between Crb2 and Cut5. (A) Pairwise yeast two-hybrid assays using the HIS3 reporter were performed between a Cut $5 \mathrm{~N}$-terminal fragment (amino acids 1-190) as bait and different Crb2 fragments as prey. Fivefold serial dilutions of cells were spotted on the indicated plates. Plates supplemented with $2.5 \mathrm{mM}$ 3-aminotriazole (3-AT) allow growth only when bait and prey interact. (B) Pairwise yeast twohybrid assays using the lac $Z$ reporter were performed between a Cut5 $\mathrm{N}$-terminal fragment (amino acids 1-190) as bait and different Crb2 fragments as prey. The values represent the means of measurements on three independent transformants. Error bars represent the standard errors of the means. $(C, D)$ Cut5 colocalizes with $\mathrm{Crb} 2$ at an $\mathrm{HO}$-induced DSB. (C) Both Crb2 and Cut5 formed HO-induced nuclear foci and their foci overlapped with each other. $(D)$ Immediately after a 36-Gy IR treatment, Crb2 nuclear foci were readily detectable, whereas Cut 5 foci occurred at much lower frequency. Only a single Z-section was captured for the fluorescence images in these panels. Bar, $5 \mu \mathrm{m}$. The strain used was LLD3672. $(E-I)$ The cut5-T401 mutation largely abolished Set9-independent HO-induced Crb2 focus formation. (E) HO-induced Crb2 foci in wild-type cells. (F) HO-induced Crb2 foci in set9d cells. $(G)$ HO-induced Crb2 foci in cut5-T401 cells. (H) The lack of HOinduced Crb2 foci in set9s cut5-T401 doublemutant cells. However, these cells did elongate upon HO-induction. (I) Quantitation of the microscopy results of the same experiment as shown in $E-H$. About $200 \operatorname{Rad} 22$ foci were counted for each strain. Strains used were LLD3650, LLD3651, LLD3673, and LLD3674. (J) The cut5-T401 mutation displayed a synthetic genetic interaction with set9s. Fivefold serial dilutions of cells were spotted on YES plates and incubated at $30^{\circ} \mathrm{C}$. IR treatment was applied directly to the plate. Plates were photographed $2 \mathrm{~d}$ later. Strains used were LLD3431, LLD3423, LLD3645, LLD3644, LLD3675, LLD3648, LLD3676, and LLD3677.

mutant cut5-T401 set9s was severely defective in HOinduced Crb2 focus formation (Fig. 6H,I), suggesting that Cut5 is important for histone modification-independent Crb2 relocalization. Even though cut5-T401 set9s mutant cells failed to form visible HO-induced Crb2 foci, they were able to arrest cell cycle progression in response to the HO-induced DSB. This checkpoint response may be due to some residual binding between $\mathrm{Crb} 2$ and mutant Cut5 that allowed a low level recruitment of Crb2.

The additive effect of combining cut5-T401 with set9s in $\mathrm{HO}$-induced Crb2 focus formation was recapitulated in DNA damage sensitivity assays. At a semipermissive temperature $\left(30^{\circ} \mathrm{C}\right)$, the double mutant of cut5-T401 and set9 $\Delta$ displayed much stronger IR and UV sensitivity than either single mutant (Fig. 6J). The IR sensitivity of set9s cut5-T401 did not quite reach the level of set9s crb2-T215A, probably because cut5-T401 did not compromise the Crb2-Cut5 interaction as completely as crb2-T215A. However, we cannot rule out the alternative possibility that $c r b 2-T 215 A$ affects more than just the Crb2-Cut5 interaction. An additive effect was also seen when crb2-T215A and cut5-T401 were combined, possibly because crb2-T215A has a more severe defect in Crb2 recruitment and cut5-T401 compromises aspects of DNA damage responses other than the Crb2-Cut5 interaction.

Taken together, our two-hybrid, microscopy, and genetic results strongly suggested a model in which a Crb2-Cut5 interaction through Crb2-T215 phosphorylation recruits $\mathrm{Crb} 2$ to sites of DNA damage independent of histone modifications.

\section{Discussion}

Checkpoint mediators are crucial for the propagation of checkpoint signals, and have been proposed to confer both sensitivity and specificity to checkpoint responses (McGowan and Russell 2004). Despite the realization that all DNA damage checkpoint mediators accumulate at DSBs, and the wide use of DNA damage-induced re- 
localization as a readout of their functions, little is known about the biological significance of the relocalization events. Also lacking is a detailed understanding of the relocalization mechanisms. In this study, we have combined localization studies with genetic analysis to reveal parallel pathways for Crb2 recruitment to DSBs. One pathway is controlled by both histone H2A phosphorylation and H4-K20 methylation, and requires the conserved Tudor and BRCT motifs in Crb2. This histone modification-dependent pathway is necessary for IRIF formation in asynchronous cells, but is dispensable for Crb2 focus formation at persistent DSBs generated by either HO or IR. The other pathway relies on an interaction between Cut5 and the $\mathrm{N}$-terminal region of $\mathrm{Crb2}$, which contains a critical CDK phosphorylation site. Disruption of either one of the pathways does not abolish Crb2 relocalization to persistent DSBs, and only results in mild loss of Crb2 functions, whereas abrogating both pathways blocks Crb2 relocalization and leads to the complete loss of Crb2 functions.

Two different histone methylation events, H3-K79 methylation and H4-K20 methylation, are required for efficient IRIF formation by 53BP1 and Crb2, respectively (Huyen et al. 2004; Sanders et al. 2004). In the case of 53BP1, a physical interaction between its Tudor repeat and methylated histone $\mathrm{H} 3$ has been observed (Huyen et al. 2004). We demonstrate here that Crb2 IRIF formation requires histone $\mathrm{H} 4-\mathrm{K} 20$ residue but not $\mathrm{H} 3-\mathrm{K} 79$. In fact, H3-K79 may not be methylated at all in fission yeast, because the fission yeast genome does not seem to encode a Dot1-like methyltransferase (our unpublished observation). Interestingly, the inverse situation applies to budding yeast. It lacks H4-K20 methylation (Schotta et al. 2004), but does have extensive H3-K79 methylation, which has been linked to DNA damage responses and G1-phase checkpoint activation (Game et al. 2005; Giannattasio et al. 2005; Wysocki et al. 2005).

In addition to the use of different Tudor-binding sites on histones, 53BP1 and Crb2 also differ in that their BRCT domains play different roles in DNA damage-induced relocalization. The BRCT domains in 53BP1 are dispensable for its IRIF formation (Iwabuchi et al. 2003; Ward et al. 2003). On the other hand, our studies have shown that the tandem BRCT repeat in Crb2 is required for both the histone modification-dependent and the histone modification-independent recruitment pathways, and is essential for the checkpoint functions of Crb2. A heterologous dimerization motif can replace the BRCT repeat for the histone modification-independent pathway, but not for the histone modification-dependent pathway, because Crb2(1-520)T215A-LZ failed to form HO-induced foci (Supplementary Fig. 3). We suspect that apart from acting as an oligomerization motif, the BRCT repeat in Crb2 either facilitates the interaction between the Tudor domain and the methylated H4 tail, or may itself bind to another ligand on the chromatin. Our genetic results indicate that $\mathrm{H} 4-\mathrm{K} 20$ methylation and $\mathrm{H} 2 \mathrm{~A}$ phosphorylation act in the same pathway to regulate Crb2, and both of them become essential in the absence of the Crb2-T215 residue. Thus, it is tempting to specu- late that phosphorylated $\mathrm{H} 2 \mathrm{~A}$, or a protein bound to phosphorylated $\mathrm{H} 2 \mathrm{~A}$, provides a binding platform for the BRCT repeat in Crb2, and that the stable association of Crb2 with chromatin only happens when both its Tudor domain and BRCT repeat have engaged with their binding targets. The need of more than one ligand for a stable interaction would also explain our failure to detect a methylation-dependent interaction between $\mathrm{Crb} 2$ and histone $\mathrm{H} 4$ peptides in in vitro binding assays (our unpublished results).

An alternative explanation for the participation of both $\mathrm{H} 2 \mathrm{~A}$ phosphorylation and H4-K20 methylation in the same Crb2 recruitment pathway is that one type of modification indirectly controls the recruitment of Crb2 through acting upstream of the other modification. In fission yeast, H4-K20 is constitutively methylated and DNA damage induced-H2A phosphorylation occurs normally in the absence of H4-K20 methylation (Sanders et al. 2004). Therefore, the modifications per se are independent of each other. Rather, it is perhaps the accessibility to the modified histone tails that is being regulated. In budding yeast, an ATP-dependent chromatin remodeling complex, Ino80, is recruited to DSBs in an H2A phosphorylation-dependent fashion (Morrison et al. 2004; van Attikum et al. 2004). Thus, one possible scenario is that $\mathrm{H} 2 \mathrm{~A}$ phosphorylation causes a chromatin conformational change, allowing Crb2 to access the methylated H4-K20 residue.

Our microscopy analyses show that histone modification mutants lose the ability to form Crb2 IRIF in asynchronous cells but are still competent in HO-induced Crb2 focus formation. We propose that Crb2 recruitment to IR-induced DSBs in asynchronous cells still occurs in these mutants, even though the level of Crb2 accumulation is not high enough to be detected by microscopy. This proposition is supported by our genetic analysis. We demonstrated that the main role of the Crb2-T215 residue is to recruit Crb2 to the sites of DNA damage. Because T215 is required for survival against IR in the absence of H2A phosphorylation or H4-K20 methylation, T215-mediated recruitment must be active in response to IR in the histone modification mutants. The cause for the higher levels of histone modification-independent Crb2 accumulation at HO-induced DSBs probably lies in the nature of the DNA damage. In an asynchronous culture, most fission yeast cells are in the G2 phase of the cell cycle, and thus have duplicated chromatids. Therefore, most IR-induced DSBs can be efficiently repaired by homologous recombination. On the other hand, when $\mathrm{HO}$ endonuclease is continuously expressed in the cells harboring an $\mathrm{HO}$ cleavage site, any attempt to repair the cleaved DNA strands by faithful religation will be thwarted by the recutting of the ligated products. Moreover, attempts at homologous recombination will be progressively crippled because both sister chromatids will eventually be cut. Therefore, HO-induced DSBs are virtually unrepairable. Attempts to repair these DSBs are likely to result in a larger extent of single-stranded DNA (ssDNA) formation than occurs during repair of IR-induced DSBs in asynchronous cells. ssDNA is a recruiting 
platform for checkpoint proteins and the amount of ssDNA is proportional to the intensity of checkpoint signaling (Lee et al. 1998; Zou and Elledge 2003; Zou et al. 2003). Thus, the stronger histone modification-independent accumulation of $\mathrm{Crb} 2$ at $\mathrm{HO}$-induced DSBs probably results from an amplified cellular response to persistent DNA damage and the ensuing accumulation of ssDNA. Consistent with this idea, we found that IR treatment of cells arrested by HU in early S phase, which presumably generated DSBs unrepairable by homologous recombination, led to a high level of Crb2 focus formation in histone modification mutants after a period of incubation in the absence of HU.

Even though the two parallel Crb2 recruitment pathways share the function of checkpoint activation, they are not completely redundant, as demonstrated by the higher-than-wild-type level DNA damage sensitivity of mutants defective in either pathway. The maintenance of a full-scale checkpoint signal probably requires both pathways acting together, as both crb2-T215A and histone modification mutants prematurely escape from IRinduced checkpoint arrest (Nakamura et al. 2004, 2005; Sanders et al. 2004). Crb2 accumulation mediated by the histone modifications is likely to rapidly spread this checkpoint mediator to a large region surrounding the DSB, and thereby contributes to signal amplification. On the other hand, the T215-mediated pathway would place Crb2 together with Cut5, which associates with sensor kinase Rad3 and PCNA-like Rad9 protein (Furuya et al. 2004). The proximity to these upstream signaling proteins may help Crb2 to act as a checkpoint mediator and thereby generate sufficient checkpoint signal even when only a small amount of Crb2 accumulates at the sites of DNA damage in IR-treated asynchronous cells.

Mammalian cells deficient in H2AX are capable of forming relatively faint 53BP1 nuclear foci during the first hour after IR treatment, and they can also redistribute 53BP1 along the tracks of laser-induced DNA damage, but these cells fail to maintain 53BP1 at sites of DNA damage (Celeste et al. 2003). In this study, we show that hta-AQ cells are capable of forming Crb2 foci at persistent DSBs. But for the following reasons, these Crb2 foci are not likely to represent the same phenomenon as the transient recruitment of 53BP1 in $\mathrm{H} 2 \mathrm{AX}$ mutant cells. First, HO-induced $\mathrm{Crb} 2$ foci in hta-AQ cells were as bright as the HO-induced foci in wild-type cells. Second, even though the long induction time required for the $\mathrm{HO}$ system precluded a precise kinetic study of focus formation, we observed that IR treatment of HU-synchronized cells appeared to result in a slower induction of Crb2 foci in histone modification-defective cells than in wild-type cells (Supplementary Fig. 4). Third and most importantly, the initial recruitment of 53BP1 relies on its interactions with methylated histones, as a point mutation in the Tudor domain completely abolished the ability of $53 \mathrm{BP} 1$ to relocalize to laser-induced DSB tracks (Bekker-Jensen et al. 2005); in contrast, Crb2 focus formation at persistent DSBs can occur independently of both the Tudor domain and the H4-K20 methylation.
A recent study showed that the $\operatorname{dot} 1 \Delta$ mutation in budding yeast partially reduces but does not abolish the accumulation of checkpoint mediator ScRad9 at an HOinduced DSB, suggesting the existence of recruitment mechanisms independent of H3-K79 methylation (Wysocki et al. 2005). Considering that ScRad9 is a CDK target (Ubersax et al. 2003), and that ScRad9-mediated checkpoint activation is severely compromised by dot $1 \Delta$ in G1 but not in G2 (Giannattasio et al. 2005; Wysocki et al. 2005), the H3-K79 methylation-independent ScRad9 recruitment pathways may be cell cycle-regulated, which is similar to our finding that a CDK phosphorylation site controls the histone modification-independent recruitment of Crb2. Therefore, the dual recruitment mechanisms for checkpoint mediators appear to be conserved in other organisms.

\section{Materials and methods}

\section{Yeast strains}

Strains used in this study are listed in Supplementary Table 1. Mutations in histone genes were created as described (Mellone et al. 2003). crb2 mutant alleles at the endogenous locus were generated by transforming crb2-D2:: $\mathrm{ura}^{+}$cells with DNA fragments containing the mutant $c r b 2$ sequences. Transformants were selected on 5-FOA plates and confirmed by PCR analysis. All strains used for DNA damage sensitivity assays and cell cycle analysis were ensured to be ura $^{+}$, because ura ${ }^{-}$cells are more resistant than ura $^{+}$cells to DNA-damaging agents such as $\mathrm{CPT}$ on plates (our unpublished observations). The strain harboring an HO cleavage site at the arg3 locus and the HO-expressing plasmid pLD102 were described previously (Du et al. 2003). For the present study, we linearized pLD102 before transformation by restriction cutting at a unique MluI site in the ars 1 region. Transformants with a single copy of plasmid integrated at the ars1 locus were selected by PCR analysis. The host strain for the two-hybrid assay was AH109 (Clontech). Cut5 was tagged by integrating at the cut5 locus a plasmid containing a C-terminal Cut5 fragment fused with CFP.

\section{Cell cycle analysis}

Synchronization was achieved by $c d c 25-22$ block release (Dunaway and Walworth 2004). Cells grown in YES medium at $25^{\circ} \mathrm{C}$ were shifted to $35.5^{\circ} \mathrm{C}$ for $2.5 \mathrm{~h}$ to arrest them in G2. They were then irradiated with a GammaCell-1000. Cells were maintained at restrictive temperature during irradiation by using a vacuuminsulated stainless-steel container (Thermos Nissan). Temperature of the medium was at $33.5^{\circ} \mathrm{C}$ at the end of a 180 -Gy irradiation. Cells were released into $25^{\circ} \mathrm{C}$ fresh medium after irradiation. Staining with 4',6-diamidine-2'-phenylindole dihydrochloride (DAPI) and Calcofluor and scoring of cells were done as described (Edwards and Carr 1997).

\section{Microscopy}

Microscopy was performed as described (Du et al. 2003). Images were acquired with a 100× 1.35-NA objective. Five Z-sections at $0.5-\mu \mathrm{m}$ intervals were projected into one image using the maximum intensity method with the softWoRx software. Unless otherwise noted, the cells with induced $\mathrm{HO}$ were observed after growing in medium without thiamine for $27 \mathrm{~h}$ at room temperature. 


\section{ChIP assay}

A published protocol was used with the following modifications (Kurdistani and Grunstein 2003). Formaldehyde was added to room-temperature cell cultures at a final concentration of $1 \%$. After 25 min cross-linking, cells were harvested and lysed with a FastPrep instrument (Qbiogene). DNA fragmentation to an average size of $1 \mathrm{~kb}$ was carried out with a Misonix Sonicator 3000. IgG Sepharose 6 Fast-Flow beads (GE Healthcare) were used to retrieve TAP-tagged Crb2. After reversing the crosslinks, QIAquick spin columns (Qiagen) were used to purify the DNA. PCR primers used were listed in Supplementary Table 2. The amounts of template DNA were titrated in pilot PCR reactions. All amplifications shown were in the linear range. PCR products were separated in 4\% NuSieve 3:1 Plus agarose Reliant gels (Cambrex). Gel photos were captured with a FluorChem imaging system (Alpha Innotech).

\section{Acknowledgments}

We thank Drs. C.H. McGowan and M.N. Boddy, and members of the Russell Laboratory for critical reading of the manuscript; the Scripps Cell Cycle Groups for discussions; anonymous reviewers for constructive comments; Robin C. Allshire for providing the strains with single copy histone H3/H4 genes; William B. Kiosses and Malcolm R. Wood at the TSRI core facility for help on microscopy; and Peiqing Sun for the use of FluorChem imager. L.-L.D. was a fellow of the Leukemia and Lymphoma Society. T.M.N. is a Sidney Kimmel Scholar. This work was funded by NIH Grant CA 77325 to P.R.

\section{References}

Bekker-Jensen, S., Lukas, C., Melander, F., Bartek, J., and Lukas, J. 2005. Dynamic assembly and sustained retention of 53BP1 at the sites of DNA damage are controlled by Mdc1/NFBD1. J. Cell Biol. 170: 201-211.

Canman, C.E. 2003. Checkpoint mediators: Relaying signals from DNA strand breaks. Curr. Biol. 13: R488-R490.

Capasso, H., Palermo, C., Wan, S., Rao, H., John, U.P., O'Connell, M.J., and Walworth, N.C. 2002. Phosphorylation activates Chk1 and is required for checkpoint-mediated cell cycle arrest. J. Cell Sci. 115: 4555-4564.

Caspari, T., Murray, J.M., and Carr, A.M. 2002. Cdc2-cyclin B kinase activity links $\mathrm{Crb} 2$ and Rqh1-topoisomerase III. Genes \& Dev. 16: 1195-1208.

Celeste, A., Fernandez-Capetillo, O., Kruhlak, M.J., Pilch, D.R., Staudt, D.W., Lee, A., Bonner, R.F., Bonner, W.M., and Nussenzweig, A. 2003. Histone H2AX phosphorylation is dispensable for the initial recognition of DNA breaks. Nat. Cell Biol. 5: 675-679.

Charier, G., Couprie, J., Alpha-Bazin, B., Meyer, V., Quemeneur, E., Guerois, R., Callebaut, I., Gilquin, B., and Zinn-Justin, S. 2004. The Tudor tandem of 53BP1: A new structural motif involved in DNA and RG-rich peptide binding. Structure (Camb.) 12: 1551-1562.

Downs, J.A. and Cote, J. 2005. Dynamics of chromatin during the repair of DNA double-strand breaks. Cell Cycle 4: 13731376.

Du, L.L., Nakamura, T.M., Moser, B.A., and Russell, P. 2003. Retention but not recruitment of $\mathrm{Crb} 2$ at double-strand breaks requires Rad1 and Rad3 complexes. Mol. Cell. Biol. 23: 6150-6158.

Du, L.L., Moser, B.A., and Russell, P. 2004. Homo-oligomerization is the essential function of the tandem BRCT domains in the checkpoint protein Crb2. J. Biol. Chem. 279: 3840938414.

Dunaway, S. and Walworth, N.C. 2004. Assaying the DNA damage checkpoint in fission yeast. Methods 33: 260-263.

Edwards, R.J. and Carr, A.M. 1997. Analysis of radiation-sensitive mutants of fission yeast. Methods Enzymol. 283: 471494.

Esashi, F. and Yanagida, M. 1999. Cde2 phosphorylation of Crb2 is required for reestablishing cell cycle progression after the damage checkpoint. Mol. Cell 4: 167-174.

Esashi, F., Mochida, S., Matsusaka, T., Obara, T., Ogawa, A., Tamai, K., and Yanagida, M. 2000. Establishment of and recovery from damage checkpoint requires sequential interactions of Crb2 with protein kinases Rad3, Chk1, and Cdc2. Cold Spring Harb. Symp. Quant. Biol. 65: 443-449.

Ferreira, M.G. and Cooper, J.P. 2004. Two modes of DNA double-strand break repair are reciprocally regulated through the fission yeast cell cycle. Genes \& Dev. 18: 2249-2254.

Furuya, K. and Carr, A.M. 2003. DNA checkpoints in fission yeast. I. Cell Sci. 116: 3847-3848.

Furuya, K., Poitelea, M., Guo, L., Caspari, T., and Carr, A.M. 2004. Chk1 activation requires Rad9 S/TQ-site phosphorylation to promote association with C-terminal BRCT domains of Rad4TOPBP1. Genes \& Dev. 18: 1154-1164.

Game, J.C., Williamson, M.S., and Baccari, C. 2005. X-ray survival characteristics and genetic analysis for nine Saccharomyces deletion mutants that show altered radiation sensitivity. Genetics 169: 51-63.

Garcia, V., Furuya, K., and Carr, A.M. 2005. Identification and functional analysis of TopBP1 and its homologs. DNA Repair (Amst.) 4: 1227-1239.

Giannattasio, M., Lazzaro, F., Plevani, P., and Muzi-Falconi, M. 2005. The DNA damage checkpoint response requires histone H2B ubiquitination by Rad6-Bre1 and H3 methylation by Dot1. J. Biol. Chem. 280: 9879-9886.

Haber, J.E. 2002. Uses and abuses of HO endonuclease. Methods Enzymol. 350: 141-164.

Harris, S., Kemplen, C., Caspari, T., Chan, C., Lindsay, H.D., Poitelea, M., Carr, A.M., and Price, C. 2003. Delineating the position of rad4+/cut5+ within the DNA-structure checkpoint pathways in Schizosaccharomyces pombe. J. Cell Sci. 116: 3519-3529.

Huyen, Y., Zgheib, O., Ditullio Jr., R.A., Gorgoulis, V.G., Zacharatos, P., Petty, T.J., Sheston, E.A., Mellert, H.S., Stavridi, E.S., and Halazonetis, T.D. 2004. Methylated lysine 79 of histone H3 targets 53BP1 to DNA double-strand breaks. Nature 432: 406-411.

Iwabuchi, K., Basu, B.P., Kysela, B., Kurihara, T., Shibata, M., Guan, D., Cao, Y., Hamada, T., Imamura, K., Jeggo, P.A., et al. 2003. Potential role for 53BP1 in DNA end-joining repair through direct interaction with DNA. J. Biol. Chem. 278: 36487-36495.

Kurdistani, S.K. and Grunstein, M. 2003. In vivo protein-protein and protein-DNA crosslinking for genomewide binding microarray. Methods 31: 90-95.

Lee, S.E., Moore, J.K., Holmes, A., Umezu, K., Kolodner, R.D., and Haber, J.E. 1998. Saccharomyces Ku70, mre11/rad50 and RPA proteins regulate adaptation to G2/M arrest after DNA damage. Cell 94: 399-409.

Lisby, M. and Rothstein, R. 2005. Localization of checkpoint and repair proteins in eukaryotes. Biochimie 87: 579-589.

Lopez-Girona, A., Tanaka, K., Chen, X.B., Baber, B.A., McGowan, C.H., and Russell, P. 2001. Serine-345 is required for Rad3-dependent phosphorylation and function of checkpoint kinase Chk1 in fission yeast. Proc. Nat1. Acad. Sci. 98: 11289-11294. 
Manke, I.A., Lowery, D.M., Nguyen, A., and Yaffe, M.B. 2003. BRCT repeats as phosphopeptide-binding modules involved in protein targeting. Science 302: 636-639.

Manolis, K.G., Nimmo, E.R., Hartsuiker, E., Carr, A.M., Jeggo, P.A., and Allshire, R.C. 2001. Novel functional requirements for non-homologous DNA end joining in Schizosaccharomyces pombe. EMBO T. 20: 210-221.

McGowan, C.H. and Russell, P. 2004. The DNA damage response: Sensing and signaling. Curr. Opin. Cell Biol. 16: 629-633.

Mellone, B.G., Ball, L., Suka, N., Grunstein, M.R., Partridge, J.F., and Allshire, R.C. 2003. Centromere silencing and function in fission yeast is governed by the amino terminus of histone H3. Curr. Biol. 13: 1748-1757.

Melo, J. and Toczyski, D. 2002. A unified view of the DNAdamage checkpoint. Curr. Opin. Cell Biol. 14: 237-245.

Mochida, S., Esashi, F., Aono, N., Tamai, K., O'Connell, M.J., and Yanagida, M. 2004. Regulation of checkpoint kinases through dynamic interaction with Crb2. EMBO J. 23: 418428

Morales, J.C., Xia, Z., Lu, T., Aldrich, M.B., Wang, B., Rosales, C., Kellems, R.E., Hittelman, W.N., Elledge, S.J., and Carpenter, P.B. 2003. Role for the BRCA1 C-terminal repeats (BRCT) protein 53BP1 in maintaining genomic stability. J. Biol. Chem. 278: 14971-14977.

Morrison, A.J., Highland, J., Krogan, N.J., Arbel-Eden, A., Greenblatt, J.F., Haber, J.E., and Shen, X. 2004. INO80 and $\gamma$-H2AX interaction links ATP-dependent chromatin remodeling to DNA damage repair. Cell 119: 767-775.

Nakamura, T.M., Du, L.L., Redon, C., and Russell, P. 2004. Histone $\mathrm{H} 2 \mathrm{~A}$ phosphorylation controls Crb2 recruitment at DNA breaks, maintains checkpoint arrest, and influences DNA repair in fission yeast. Mol. Cell. Biol. 24: 6215-6230.

Nakamura, T.M., Moser, B.A., Du, L.L., and Russell, P. 2005. Cooperative control of Crb2 by ATM family and Cdc2 kinases is essential for the DNA damage checkpoint in fission yeast. Mol. Cell. Biol. 25: 10721-10730.

Pryde, F., Khalili, S., Robertson, K., Selfridge, J., Ritchie, A.M., Melton, D.W., Jullien, D., and Adachi, Y. 2005. 53BP1 exchanges slowly at the sites of DNA damage and appears to require RNA for its association with chromatin. J. Cell Sci. 118: 2043-2055.

Rigaut, G., Shevchenko, A., Rutz, B., Wilm, M., Mann, M., and Seraphin, B. 1999. A generic protein purification method for protein complex characterization and proteome exploration. Nat. Biotechnol. 17: 1030-1032.

Saka, Y., Fantes, P., Sutani, T., McInerny, C., Creanor, J., and Yanagida, M. 1994. Fission yeast cut5 links nuclear chromatin and $M$ phase regulator in the replication checkpoint control. EMBO J. 13: 5319-5329.

Saka, Y., Esashi, F., Matsusaka, T., Mochida, S., and Yanagida, M. 1997. Damage and replication checkpoint control in fission yeast is ensured by interactions of Crb2, a protein with BRCT motif, with Cut5 and Chk1. Genes \& Dev. 11: 33873400.

Sanders, S.L., Portoso, M., Mata, J., Bahler, J., Allshire, R.C., and Kouzarides, T. 2004. Methylation of histone H4 lysine 20 controls recruitment of Crb2 to sites of DNA damage. Cell 119: 603-614.

Schotta, G., Lachner, M., Sarma, K., Ebert, A., Sengupta, R., Reuter, G., Reinberg, D., and Jenuwein, T. 2004. A silencing pathway to induce $\mathrm{H} 3-\mathrm{K} 9$ and $\mathrm{H} 4-\mathrm{K} 20$ trimethylation at constitutive heterochromatin. Genes \& Dev. 18: 1251-1262.

Soulier, J. and Lowndes, N.F. 1999. The BRCT domain of the $S$. cerevisiae checkpoint protein Rad9 mediates a Rad9-Rad9 interaction after DNA damage. Curr. Biol. 9: 551-554.
Stucki, M. and Jackson, S.P. 2004. Tudor domains track down DNA breaks. Nat. Cell Biol. 6: 1150-1152.

Tommasino, M. and Maundrell, K. 1991. Uptake of thiamine by Schizosaccharomyces pombe and its effect as a transcriptional regulator of thiamine-sensitive genes. Curr. Genet. 20: 63-66.

Ubersax, J.A., Woodbury, E.L., Quang, P.N., Paraz, M., Blethrow, J.D., Shah, K., Shokat, K.M., and Morgan, D.O. 2003. Targets of the cyclin-dependent kinase Cdk1. Nature 425: 859-864.

van Attikum, H. and Gasser, S.M. 2005. The histone code at DNA breaks: A guide to repair? Nat. Rev. Mol. Cell Biol. 6: 757-765.

van Attikum, H., Fritsch, O., Hohn, B., and Gasser, S.M. 2004. Recruitment of the INO80 complex by H2A phosphorylation links ATP-dependent chromatin remodeling with DNA double-strand break repair. Cell 119: 777-788.

Vidanes, G.M., Bonilla, C.Y., and Toczyski, D.P. 2005. Complicated tails: Histone modifications and the DNA damage response. Cell 121: 973-976.

Wang, B., Matsuoka, S., Carpenter, P.B., and Elledge, S.J. 2002. 53BP1, a mediator of the DNA damage checkpoint. Science 298: $1435-1438$.

Ward, I.M., Minn, K., Jorda, K.G., and Chen, J. 2003. Accumulation of checkpoint protein 53BP1 at DNA breaks involves its binding to phosphorylated histone H2AX. J. Biol. Chem. 278: 19579-19582.

Wysocki, R., Javaheri, A., Allard, S., Sha, F., Cote, J., and Kron, S.J. 2005. Role of dot1-dependent histone $\mathrm{H} 3$ methylation in G1 and S phase DNA damage checkpoint functions of Rad9. Mol. Cell. Biol. 25: 8430-8443.

Yu, X., Chini, C.C., He, M., Mer, G., and Chen, J. 2003. The BRCT domain is a phospho-protein binding domain. Science 302: 639-642

Zou, L. and Elledge, S.J. 2003. Sensing DNA damage through ATRIP recognition of RPA-ssDNA complexes. Science 300: $1542-1548$.

Zou, L., Liu, D., and Elledge, S.J. 2003. Replication protein Amediated recruitment and activation of Rad17 complexes. Proc. Natl. Acad. Sci. 100: 13827-13832. 


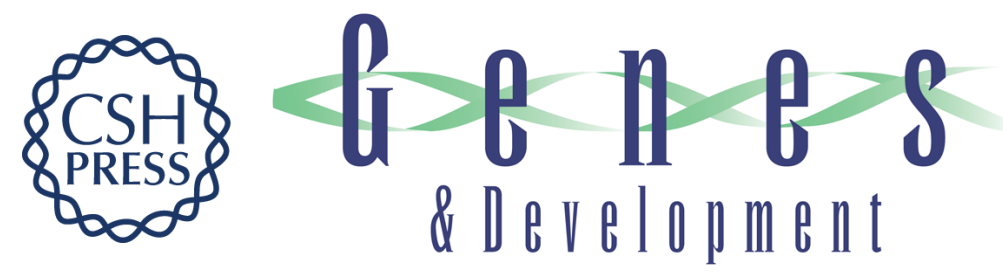

\section{Histone modification-dependent and -independent pathways for recruitment of checkpoint protein Crb2 to double-strand breaks}

Li-Lin Du, Toru M. Nakamura and Paul Russell

Genes Dev. 2006, 20:

Access the most recent version at doi:10.1101/gad.1422606

Supplemental http://genesdev.cshlp.org/content/suppl/2006/06/01/20.12.1583.DC1
Material

References This article cites 56 articles, 28 of which can be accessed free at:

http://genesdev.cshlp.org/content/20/12/1583.full.html\#ref-list-1

License

Email Alerting Receive free email alerts when new articles cite this article - sign up in the box at the top

Service right corner of the article or click here.

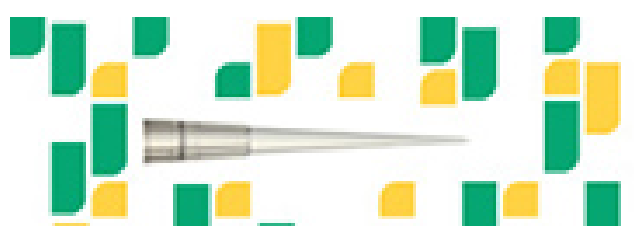

Focused on your science. 